\title{
LES of flow in the street canyon
}

\author{
Vladimír FUKA ${ }^{*}$,Josef BRECHLER ${ }^{*}$
}

\begin{abstract}
Results of computer simulation of flow over a series of street canyons are presented in this paper. The setup is adapted from an experimental study by [4] with two different shapes of buildings. The problem is simulated by an LES model CLMM (Charles University Large Eddy Microscale Model) and results are analysed using proper orthogonal decomposition and spectral analysis. The results in the channel (layout from the experiment) are compared with results with a free top boundary.
\end{abstract}

\section{INTRODUCTION}

The coherent structures in the turbulent flow carry a substantial part of the turbulent kinetic energy (TKE) and determine the form of the flow. They can be examined by the proper orthogonal decomposition (POD) [6]. The inputs for the POD analysis are the time and space dependent fields of the state variables (e.g. wind velocity). The decomposition is performed on series of snapshots of the flow. The fields can be results of measurement or simulation.

An experimental study by Kellnerová et al.[4] studied flow in a series of parallel street canyons. This paper presents computation of a similar setup. The main differences are the cyclic inflow and outflow conditions used for the simulation. Also the efect of the upper wall used in the experiment is examined.

\section{Numerical MOdEL}

The simulations were performed using the model CLMM (Charles University Microscale Model [3]) which solves the incompressible Navier-Stokes equations. The small turbulent motions are modelled using large eddy simulation (LES). The equations are solved on a staggered orthogonal grid. The projection method is used to couple the momentum equations and the continuity equation. The Vreman [7] model is used as a closure for computation of the subgrid stresses. It is an algebraic eddy viscosity model which does not suffer from excessive dissipation in laminar areas and near solid walls. Because of the orthogonal grid the immersed boundary method is used to enforce the correct geometry of the obstacles [5] [1]. The subgrid stresses near solid walls were computed by a wall model using a wall function.

\section{SimULATED PROBLEM}

The experimental setup by Kellnerová et al. [4] consisted of a square wind channel $25 \times 25 \mathrm{~cm}$ in crosssection approximately $3 \mathrm{~m}$ long with a series of parallel street canyons with two types of buildings. The first building shape was a building with a flat roof $\mathrm{H}=5 \mathrm{~cm}$ wide and high. The other type had pitched roof that was made by cutting the first type of building at $3 \mathrm{~cm}$ height. The street was $5 \mathrm{~cm}$ wide in both cases. The geometry is apparent from figures in the following parts.

In our simulation we used a $2 \mathrm{~m}$ long domain with the same cross-section and building dimensions, i.e. we had 20 parallel buildings in the domain. For the inflow and outflow we used cyclic boundary conditions. That means we had periodic row of 20 street canyons.

The flow was driven by a constant pressure gradient that was kept the same value in both cases. That caused a little difference in the wind speed in the centre of the channel. The maximum time-averaged wind speed in the channel with the flat roof was $6.2 \mathrm{~ms}^{-1}$, whereas for pitched roofs it was $5.2 \mathrm{~ms}^{-1}$.

The simulation of the channel was carried out on a uniform grid with $640 \times 97 \times 97$ control volumes. We also performed simulations with shorter domain, but the flow was then dominated by a wave with the wavelength equal to the length of the domain.

The flow was also simulated in a hypothetical configuration with a free-slip upper boundary condition in $50 \mathrm{~cm}$ height with $640 \times 97 \times 193$ control volumes in the uniform grid.

\footnotetext{
* Charles University in Prague, Faculty of Mathematics and Physics, Department of Meteorology and Environment Protection, V Holešovičkách 2, Prague 8, 180 00, vladimir.fuka@mff.cuni.cz, josef.brechler@mff.cuni.cz
} 
At the surface of the street canyons a $2 \mathrm{D}$ source of a passive scalar is placed. Its release rate is $1 \mathrm{~m}^{-2} \mathrm{~s}^{-1}$

\section{Proper ORTHOGONAL DECOMPOSITION}

The proper orthogonal decomposition is a method for getting a new orthogonal basis of the state space, in which the individual base vectors (so called modes) explain the largest possible part of the variability of the time dependent system. The state of the system in each time can be written as a linear combination of the modes

$$
q_{j}-\bar{q}=\sum_{k} c_{j k} \phi_{k}
$$

where $q_{j}$ are snapshots of the state vector at different times, $\bar{q}$ is the average state, $\phi_{k}$ are the POD modes and POD coefficients. The modes are solution to an optimization problem, to maximize a functional

$$
\max _{q_{1}, \ldots, q_{2}} \sum_{i=1}^{l} \sum_{j=1}^{n}\left|<q_{j}, \phi_{i}>\right|^{2}, \quad \text { such that }<\phi_{i}, \phi_{j}>=\delta_{i j} .
$$

In the context of turbulent flow we can interpret POD modes with lower index also as structures containing the most of turbulent kinetic energy (TKE). We used Fortran program SVD_BASIS by John Burkardt to solve (1). It is based on singular value decomposition (SVD)[2].

\section{REsULtS}

The simulated time interval was $10 \mathrm{~s}$ long in all geometrical configurations and the last seven seconds were used to produce snapshots and other statistics. The time averaged flow in the both types of street canyon is in Fig. 1. We stored $100002 \mathrm{D}$ snapshots of the flow (all three velocity components) and passive scalar concentrations at the central plane $x z$. We computed the POD modes on a section of the plane covering a close proximity of one selected canyon. The first 3 modes for the pitched roof case are plotted in Fig. 2. They have a similar shape to those computed by Kellnerová et al.[4] from their PIV measurements. However, at the time of writing, we didnât have access to the exact numerical data from the experiment yet.
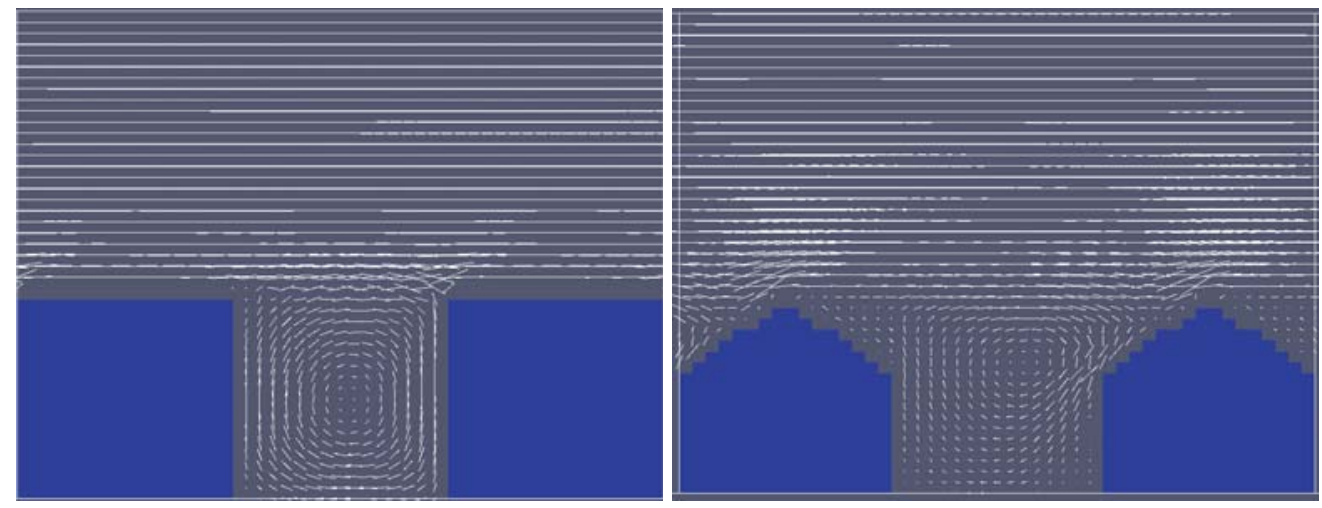

Figure 1: The time averaged flow in the street canyon for the flat roofs (left) and the pitched roofs (right).

From the computed flow fields themselves, and also from the POD analysis, it was clear that the flow is more complex in the case of pitched roofs. In the case of flat roofs the vortices separated at the lee side of the building continue mostly directly to the edge of the building and form a quasi-steady shear layer at the top of the canyon. Also the main vortex in the street canyon itself is more stable and is well pronounced almost at all times. This is not true in the case of pitched roofs where a recirculation zone behind upwind building's roof forms but is not totally stable. The vortices detached from the top edge of the building continue in different directions and impinge on the next building in different positions or even continue above it. These findings are consistent with the experimental study [4]. The following analysis will mostly concerns the pitched roofs. 

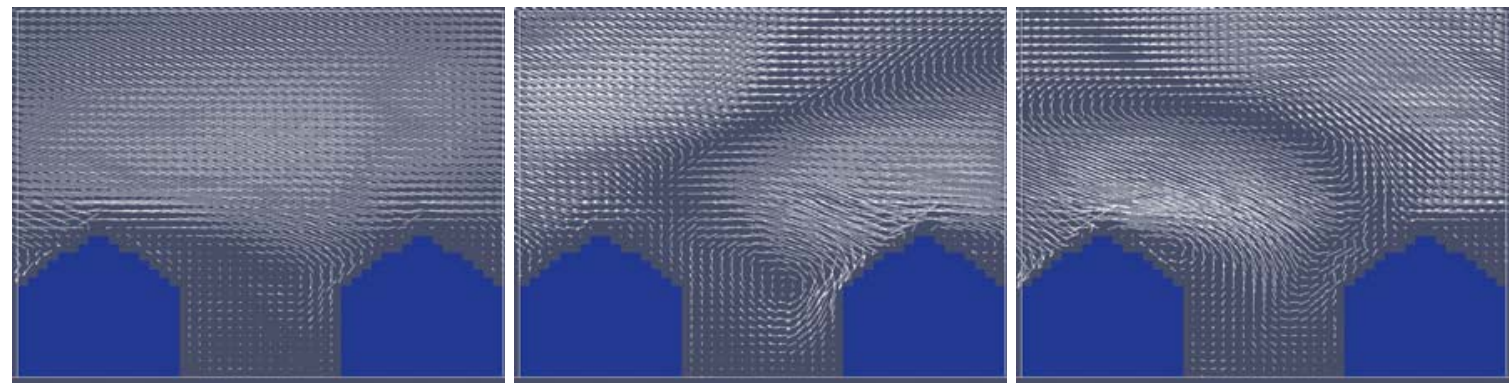

Figure 2: The first 3 POD modes for the pitched roofs.

We also performed Fourier spectral analysis of the velocity component time series in the middle of the canyon at the roof level height. The spectra are plotted in Fig. 3. There is a very obvious distinction between the two roof shapes. For the flat roofs, the inertial range is not well developed. The possible cause of this is the fact that the inertial range begins at higher frequencies, which are close to the subgrid scale. Also turbulence in the canyon is very far from theoretical case that assumes the Taylor's frozen turbulence hypothesis. In the case of pitched roofs, the spectra show a clear inertial range between approximately $10 \mathrm{~Hz}$ and $200 \mathrm{~Hz}$. The high frequency end of the resolved inertial range is caused by the limited resolution. The computational grid cannot resolve motions smaller than $6 \mathrm{~mm}$ and the spatial scale and time scale of turbulence are closely connected. The low dominating frequencies of the flow are probably affected by the cyclic boundary conditions which prefer frequencies that are a multiple of the mean advection velocity above the roof level which is roughly $5.6 \mathrm{~ms}^{-1}$ for flat roofs and $4.5 \mathrm{~ms}^{-1}$ for pitched roofs divided by the domain length $(2 \mathrm{~m})$.
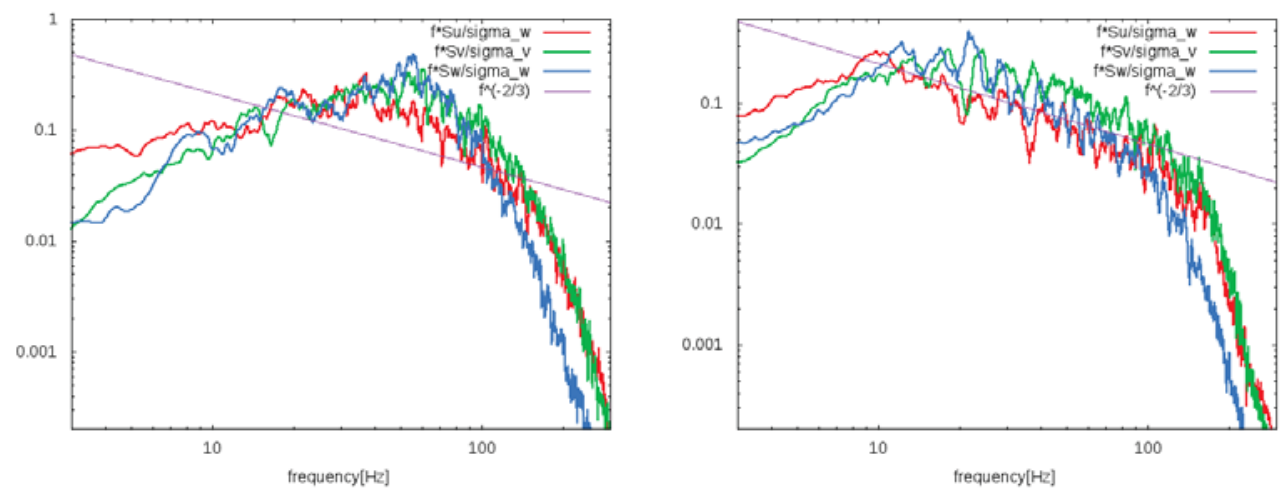

Figure 3: The (filtered) spectra of velocity components at the centre of the channel at the height $\mathrm{z}=\mathrm{H}$ for flat roofs (left) and pithed roofs (right).

Another task performed was to compute the Fourier spectra of the POD coefficients of first three modes (Fig. 4). They contain less high frequency parts, than the velocity components themselves, as expected. Some of the peaks are common among the modes, but the first mode contains considerably more low frequencies. The same argument about spoiling the frequency spectrum because of the boundary conditions applies here. The coherence of the modes can be seen in a better way by the cross correlation functions in Fig. 5. The peaks in the cross correlation between first four consecutive modes are above 0.4 and in the case of the correlation between modes 3 and 4 above 0.5. This means that the POD modes are not independent in their dynamics. In the graph of time series of the coefficients in Fig. 6 one can see that the peaks of mode 1 coefficient are often followed by the peak of mode 2 . This feature was also found in the experiment and Kellnerová et al. interpret this as the mode 1 bringing more kinetic energy to the canyon by strengthening the recirculation behind the upwind roof and the mode 2 responding by strengthened main vortex. For the other consecutive modes, the cross-correlation is opposite, i.e. the mode with lower number is lagged behind the mode with higher number. 


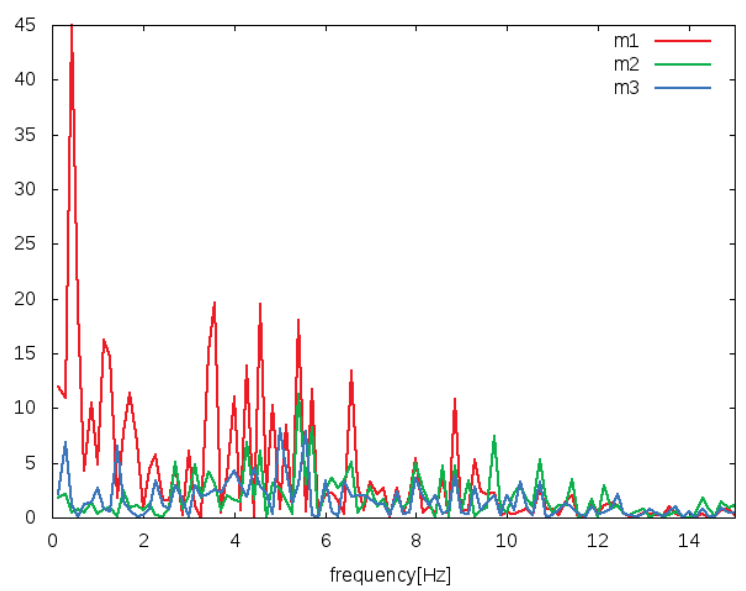

Figure 4: The spectra of POD time coefficients for first 3 modes.

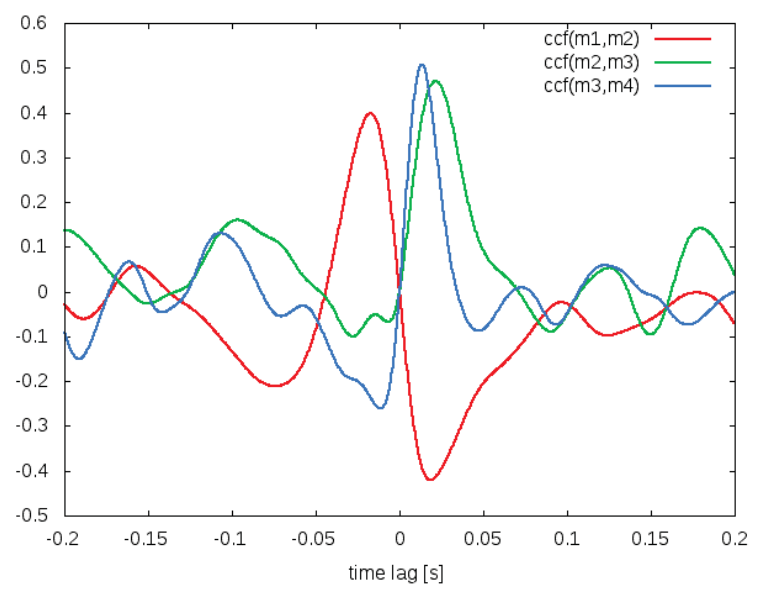

Figure 5: The cross correlation functions between the first four consecutive POD modes for pitched roofs.

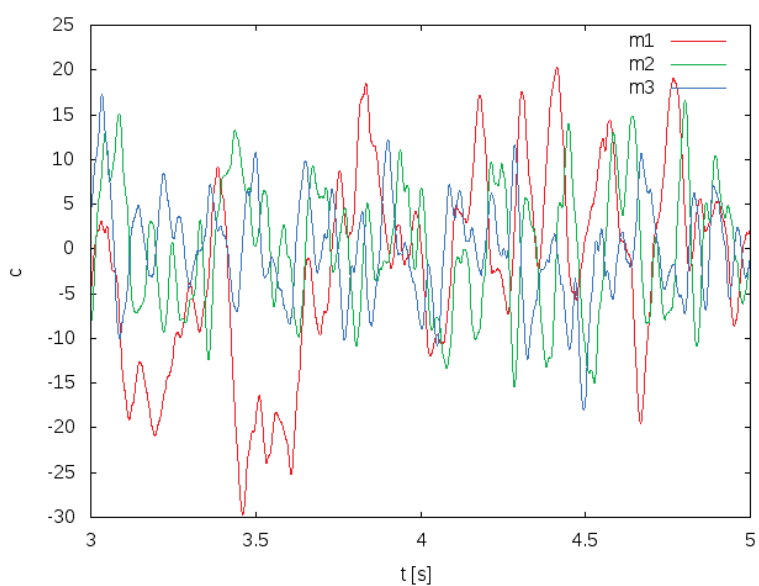

Figure 6: A part of the time series of POD coefficients for first 3 modes for pitched roofs. 
The spanwise velocity fluctuations form interesting features of the flow, that couldnât have been measured in the wind channel. In our simulations they produce ramp like features with dimension comparable to the building dimension, so that they are the prevailing coherent structures above the canopy. A typical example of their appearance is in Fig. 7. In the computation with the higher positioned free-slip top boundary they reach approximately the height of $3 \mathrm{H}$. Finally, in Fig. 8 the vortical structures in the flow are visualized using the $\lambda_{2}$ criterion. They tend to dominate the flow above the canopy. Because they are smaller in size than the structures of spanwise velocity, they are probably somehow organized but this has not been investigated yet.

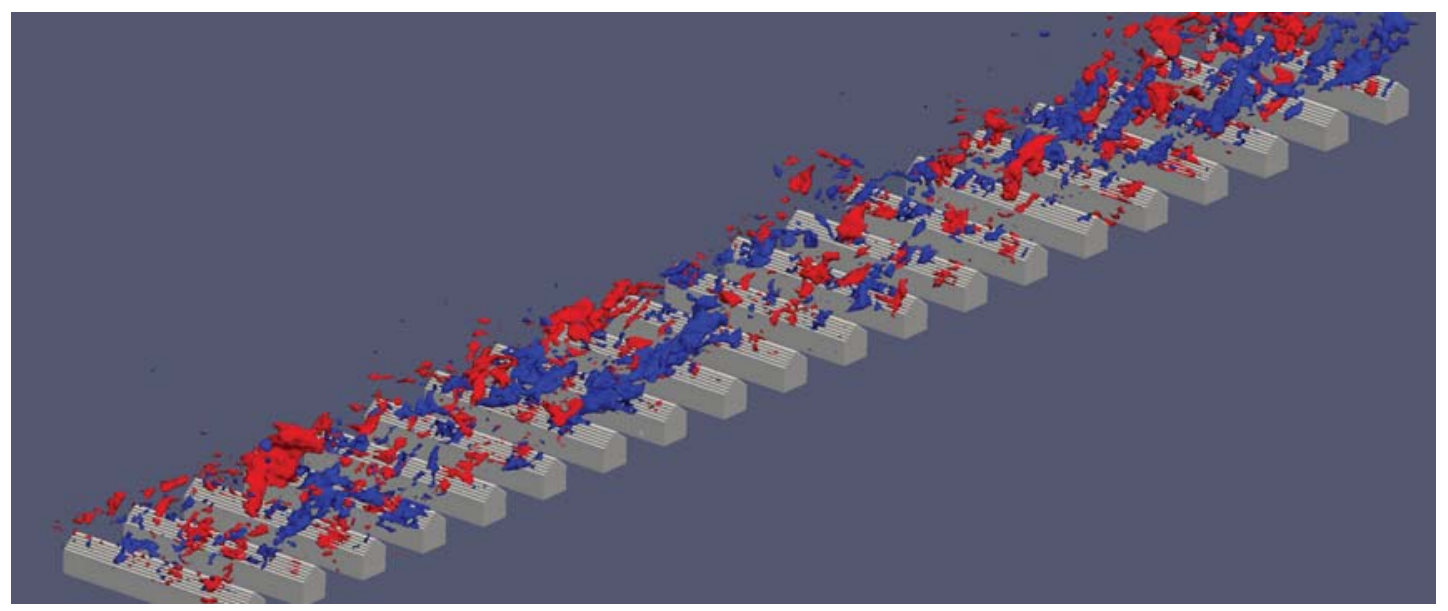

Figure 7: The isocontours of the spanwise velocity component $-1 \mathrm{~ms}^{-1}$ (blue) and $+1 \mathrm{~ms}^{-1}$ (red).

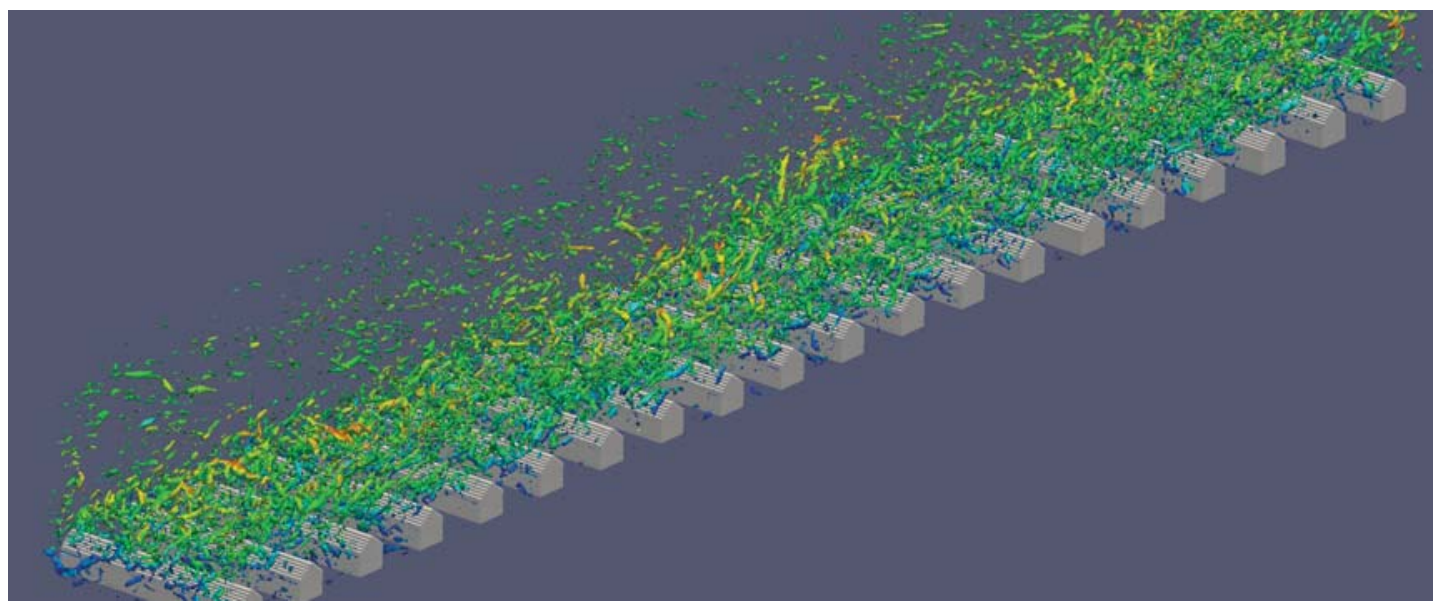

Figure 8: The turbulent vortices visualised using the $\lambda_{2}$ criterion.

We were concerned about the effect of the upper wall of the channel, which obviously causes a different velocity profile. We therefore computed a simulation with the same geometry and pressure gradient, but with a two times higher domain and a free-slip boundary (with a sponge layer below) at the top. As seen in Fig. 9 the logarithmic region is much smaller in the channel configuration. Luckilly, the flow in the canyons and their vicinity is not so much affected (including the POD modes in Fig. 10).

As a last test we performed also a preliminary computation of passive scalar dispersion from the canyon. We used contant rate source at the complete bottom of the canyon with the release rate of $Q=1 \mathrm{~m}^{-2} \mathrm{~s}^{-1}$. In Figs. 11 and 12 are depicted time-averaged concentrations for the two geometries in the channel. One can see that due to enhanced mixing, there are smaller concentrations in the street canyon with the pitched roofs. In the flat roofs case there is a trace of plume continuing at the the top of the canyon 


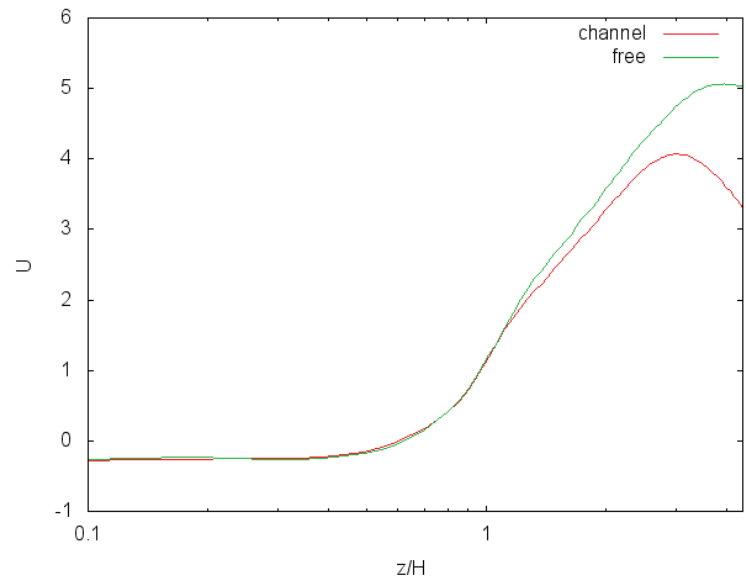

Figure 9: Comparison of the time averaged profiles of horizontal velocity component in the middle of the canyon for the channel and free-slip top configurations.
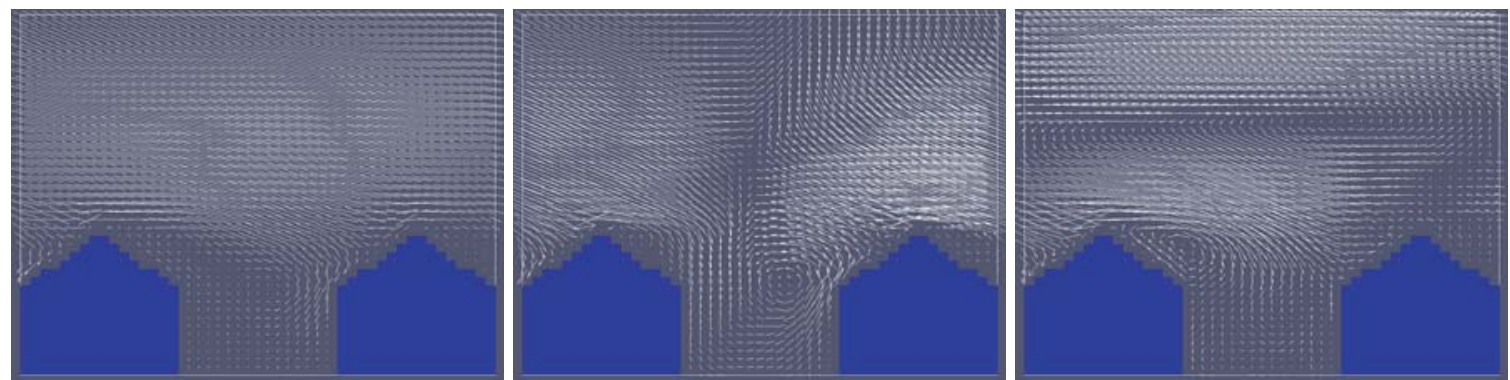

Figure 10: The first 3 POD modes for the pitched roofs in configuration with a free-slip top boundary. 
and remainng in the canyon, thus not being well mixed with the air above.

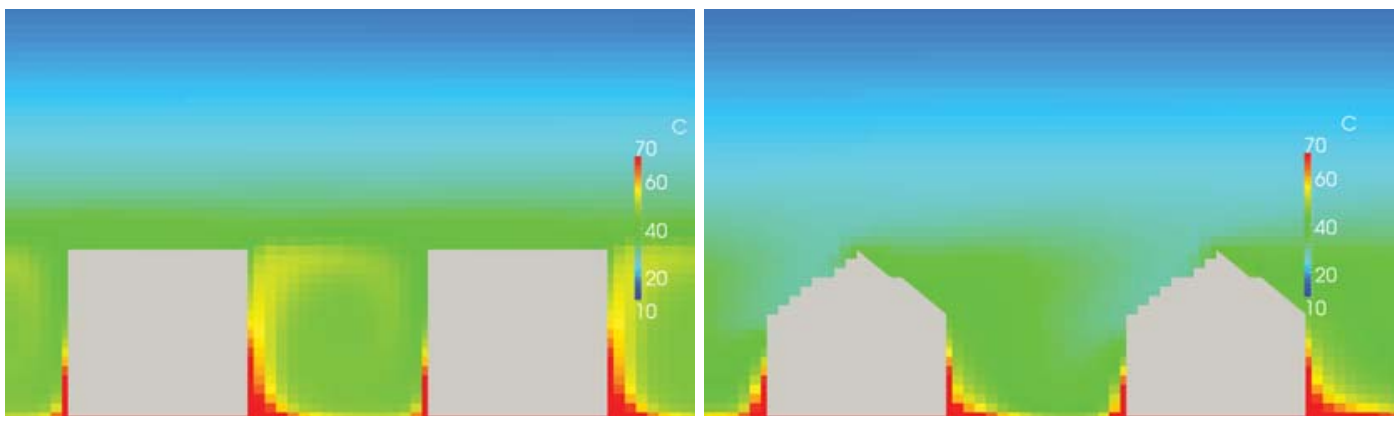

Figure 11: The nondimensional concentrations of the passive scalar in the channel the two geometries.

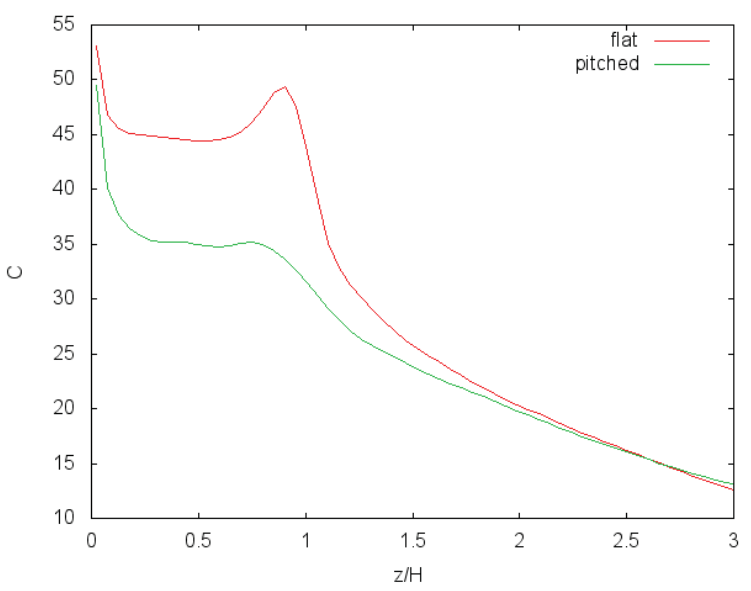

Figure 12: The profiles of the nondimensional concentrations of the passive scalar in the middle of the street canyons for the two geometries.

\section{Conclusions}

We performed a large eddy simulation of the flow in a channel with parallel street canyon based on an experiment. We were able to compare only qualitative agreement between our results and experiment. Probably a higher resolution should be desired as we had only 16 grid cells in the street canyon. With our present hardware we would have to sacrifice number of the canyons in the periodic domain, which would lead to a strong limitation of reliability of the spectral properties of the flow. The flow in the canyon itself seems not to be affected by the domain length so much in our preliminary tests. We also performed with the free top boundary condition positioned higher above the buildings to assess the effect of blockage. It proved that the logarithmic layer above the buildings was strongly suppressed in the channel configuration, but the flow inside the canyons and just above them was not affected so much. The preliminary tests of passive scalar dispersion showed differences in ventilation of the two shapes of the street canyons, with larger concentrations found in the canyons with flat roofs.

\section{ACKNOWLEDGMENTS}

The work was supported by the Czech Ministry of Education, Youth and Sports under the framework of research plan MSM0021620860. 


\section{REFERENCES}

[1] Bodnár T., Beneš L., Kozel K., Numerical simulation of flow barriers in complex terrain, Nuovo Cimento Della Societa Italiana Di Fisica C 31, pp. 619-632, 2008

[2] Burkardt J., Gunzburger M., Lee, H.-C. , Centroidal Voronoi Tessellation-Based Reduced-Order Modelling of Complex Systems, SIAM Journal on Scientific Computing, 28, 2, pages 459-484, 2006.

[3] Fuka V. and Brechler J., Large Eddy Simulation of the Stable Boundary Layer, In: Fort, J Fürst, J Halama, R Herbin and F Hubert (eds.), Finite Volumes for Complex Applications VI Problems \& Perspectives, Springer Proceedings in Mathematics, Volume 4, Part 1, 485-493, 2011

[4] Kellnerová R., Kukačka L., Uruba V., Antoš P., Odin J., Jaňour Z., Wavelet and POD Analysis of Turbulent Flow Within Street Canyon, Experimental fluid mechanics 2010. Jičín (Czech Republic), 2010

[5] Kim J., Kim D., Choi H. An Immersed-Boundary Finite-Volume Method for Simulations of Flow in Complex Geometries, J. Comp. Phys. 171. pp. 132-150, 2001

[6] Sirovich L. Turbulence and dynamics of coherent structures. Part I: coherent structures, Q. Appl. Math. 45. pp. 561-571., 1987

[7] Vreman A., An eddy-viscosity subgrid-scale model for turbulent shear flow: Algebraic theory and applications, Phys. Fluids 16. pp. 3670-3681, 2004 